\title{
Atomic, Molecular and Cluster Science with the Reaction Microscope Endstation at FLASH2
}

\author{
Severin Meister ${ }^{1, *(\mathbb{D}}$, Hannes Lindenblatt ${ }^{1}\left(\mathbb{D}\right.$, , Florian Trost ${ }^{1}\left(\mathbb{D}\right.$, Kirsten Schnorr $^{2}{ }^{(0)}$, \\ Sven Augustin ${ }^{2}\left(\mathbb{D}\right.$, Markus Braune ${ }^{3}\left(\mathbb{D}\right.$, Rolf Treusch $^{3}{ }^{\circ}$, Thomas Pfeifer ${ }^{1}{ }^{(0)}$ \\ and Robert Moshammer ${ }^{1}$ \\ 1 Max-Planck-Institute for Nuclear Physics, 69117 Heidelberg, Germany; \\ hannes.lindenblatt@mpi-hd.mpg.de (H.L.); trost@mpi-hd.mpg.de (F.T.); \\ thomas.pfeifer@mpi-hd.mpg.de (T.P.); r.moshammer@mpi-hd.mpg.de (R.M.) \\ 2 Paul Scherrer Institute, 5232 Villigen, Switzerland; kirsten.schnorr@psi.ch (K.S.); sven.augustin@psi.ch (S.A.) \\ 3 Deutsches Elektronen-Synchrotron, 22607 Hamburg, Germany; markus.braune@desy.de (M.B.); \\ rolf.treusch@desy.de (R.T.) \\ * Correspondence: severin.meister@mpi-hd.mpg.de; Tel.: +49-6221-516-438
}

Received: 31 March 2020; Accepted: 21 April 2020; Published: 24 April 2020

\begin{abstract}
The reaction microscope (REMI) endstation for atomic and molecular science at the free-electron laser FLASH2 at DESY in Hamburg is presented together with a brief overview of results recently obtained. The REMI allows coincident detection of electrons and ions that emerge from atomic or molecular fragmentation reactions in the focus of the extreme-ultraviolet (XUV) free-electron laser (FEL) beam. A large variety of target species ranging from atoms and molecules to small clusters can be injected with a supersonic gas-jet into the FEL focus. Their ionization and fragmentation dynamics can be studied either under single pulse conditions, or for double pulses as a function of their time delay by means of FEL-pump-FEL-probe schemes and also in combination with a femtosecond infrared (IR) laser. In a recent upgrade, the endstation was further extended by a light source based on high harmonic generation (HHG), which is now available for upcoming FEL/HHG pump-probe experiments.
\end{abstract}

Keywords: atom; molecule; REMI; endstation; FLASH

\section{Introduction}

The invention of intense femtosecond infrared (IR) lasers [1] led to a new era in atomic and molecular physics, namely the devolopment of femtochemistry [2]. Today it is often mentioned as one part of a much larger multi-disciplinary field called ultra-fast physics [3]; the observation of light-induced dynamics in small quantum systems on their natural time scale in the range of femto- or even attoseconds. In many cases a first "pump" pulse triggers certain dynamics, while a delayed "probe" pulse is used to interrogate the system at a later point in time. This way, the temporal evolution of any specific process under investigation like molecular break-up [4], charge migration [5,6] or isomerization [7] within a molecule, to just name a few, can be observed on their natural time scale by using lasers with correspondingly short pulse lengths in the range of a few femtoseconds. A small number of different technologies exists to produce such short laser-like or coherent light pulses. Depending on the specific application each of them comes with characteristic advantages and drawbacks.

Free-electron lasers (FELs) are large-scale electron-accelerator-based machines. They cover a huge range of photon energies, from the microwave [8] up to the hard X-ray regime [9], with unsurpassed light intensities for photon energies above $10 \mathrm{eV}[3,10]$. However, most FELs are operated at comparably 
low repetition rates (typically below $1 \mathrm{kHz}$ ) and are providing light pulses that are usually only partly coherent. In contrast, conventional femtosecond lasers in the visible or IR-range provide pulses with high repetition rates, high intensities and excellent coherence properties [1,11,12]. However, their spectral range is limited to photon energies of a few $\mathrm{eV}$ only. This limitation is resolved with high harmonic-generation (HHG) sources [13,14], where high-intensity femtosecond IR laser pulses are sent into a gaseous target to drive a non-linear light-atom interaction. This leads to the emission of frequency up-converted (higher harmonic) radiation [15] with unique temporal and spectral properties. HHG sources are ideally suited to produce extremely short pulse durations of only few tens of attoseconds [16] with photon energies of up to several hundred eV [17]. However, these sources are limited, due to the low conversion efficiencies, to light intensities that are orders of magnitude lower than those delivered by modern XUV or X-ray free-electron lasers [18].

For studies of time-dependent processes in small quantum systems it is of great advantage if an experimental station allows high flexibility with respect to the usage of one or a combination of the above mentioned light sources, which are ideally adapted to the given reaction dynamics. With the reaction microscope (REMI) endstation at FLASH2, we implemented this to a large extent. In its present stage, it routinely allows FEL-based pump-probe experiments with atomic, molecular or cluster targets in the gas phase. The experimental station consists of a multi-particle imaging spectrometer for electrons and ions (REMI), the FEL beamline with integrated split-and-delay and focussing optics for FEL pulse-pair creation [19], an IR laser for femtosecond FEL-pump laser-probe experiments, and a laser-driven HHG source for two-color XUV pump-probe measurements. A manuscript about technical details and benchmarks of the REMI endstation can be found in [19]. In contrast, this review provides with information about physical quantities and experimental schemes which are accessible by the REMI endstation. The following manuscript describes in Section 2 the experimental capabilities of the REMI endstation at FLASH2 and a selection of published results (Sections 3.2 and 3.3) as well as unpublished results (Section 3.1) is briefly discussed. Concluding statements are given in Section 4.

\section{Experimental Setup}

A design drawing of the REMI endstation with its main components is shown in Figure 1. It is installed at beamline FL26 in the experimental hall of FLASH2. Along the beamline, several diagnostic and beam shaping elements like imaging screens, apertures and slits can be inserted into the beampath. In the subsequent section the FEL beam impinges on the split-delay and focussing optics. The former consists of two planar mirrors that are stacked one upon the other to cut the incoming beam geometrically into two co-propagating half-moon shaped beams. By moving one planar mirror with respect to the other, a small change of the path length can be introduced, which leads to a temporal delay of one of the two pulses. The delay range is $\pm 2500 \mathrm{fs}$, with a resolution better than $1 \mathrm{fs}$. The ellipsoidal focussing mirror has a focal length of $1 \mathrm{~m}$. It creates a demagnified image of the FEL source-point (the FEL undulator is about $85 \mathrm{~m}$ upstream) in the center of the REMI chamber. Wavefront-sensor measurements yield an optimized focus size of about $5 \mu \mathrm{m}$ diameter [19]. The split and delay function is optional, as each planar mirror is large enough to reflect the full FEL beam on its own surface, if centered in the beam. With the given mirror coating and the grazing incidence geometry, the overall transmission is better than $50 \%$ for all photon energies up to $180 \mathrm{eV}$. A more detailed description of the setup with limits and benchmarks can be found in Ref. [19].

\subsection{The Free-Electron Laser FLASH2}

The XUV free-electron laser FLASH is the first short-wavelength FEL and went into user operation in 2005 at DESY in Hamburg [20]. FLASH2 is an extension of FLASH that came into operation in 2016 ([21,22]). It shares the electron accelerator with FLASH1, but features its own set of undulators. Compared to FLASH1, the FLASH2 undulators have variable gaps allowing a fast change or a scan of the photon wavelength over a large range. Due to the shared accelerator and the fixed undulator gaps at FLASH1, the accessible wavelengths of FLASH2 depend on the delivered wavelength at FLASH1. 
It ranges from the delivered wavelength at FLASH1 to approximately three times this wavelength, for a given accelerator setup [22]. Lasing in the undulator is achieved by self-amplified spontaneous emission (SASE), which results in short (10 to $200 \mathrm{fs}$ [23]) and intense FEL pulses at the expense of a limited pulse-to-pulse stability with respect to intensity and spectral properties, when compared to optical lasers. FLASH2 delivers photon energies in a range from 14 to $310 \mathrm{eV}$. For light in the REMI, the upper limit is $180 \mathrm{eV}$ due to the dropping reflectivity of carbon-coated mirrors under $8^{\circ}$. FLASH generates electron bunches, and hence photon pulses, in a burst mode. The pulse pattern consists of short pulse trains repeating at $10 \mathrm{~Hz}$. Each pulse train has a length of typically $500 \mu \mathrm{s}$ and comprises up to 500 pulses, resulting in a maximum intra-train repetition rate of $1 \mathrm{MHz}$. Averaged over time, a maximum repetition rate of $5 \mathrm{kHz}$ can be delivered.

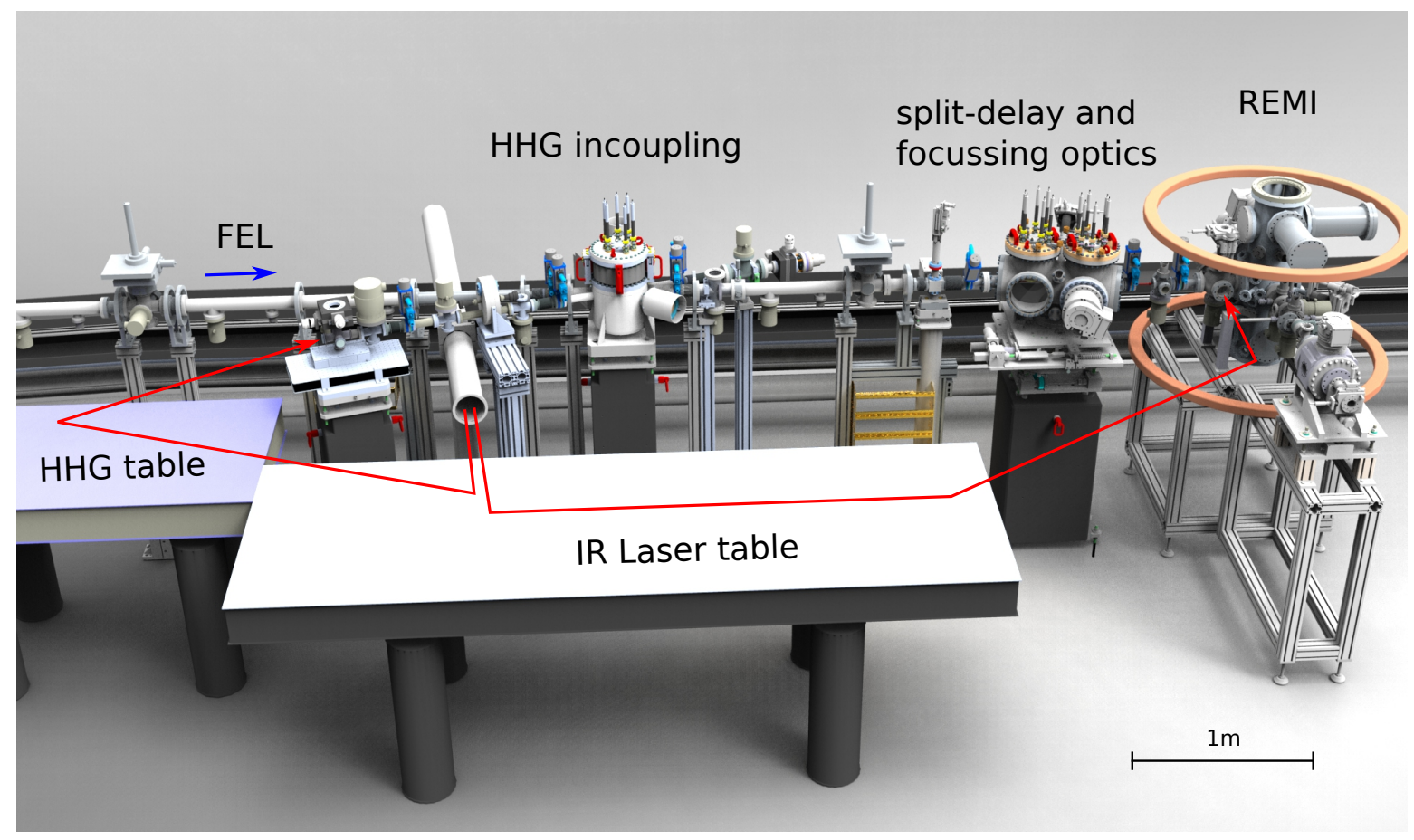

Figure 1. Overview of the reaction microscope (REMI) endstation. The free-electron laser (FEL) is delivered from the left, while the infrared (IR) laser is delivered from the rear, crossing below the beamline. The IR is coupled into the beamline right before the REMI with a holey mirror. The HHG (high harmonic-generation) light is coupled by a grazing angle about $3 \mathrm{~m}$ upstream (indicated by the two red arrows).

\subsection{IR Laser}

For XUV-IR pump-probe experiments, an $800 \mathrm{~nm}$ OPCPA (optical parametric chirped pulse amplification) IR-laser is operated by DESY. Pulse energies up to $500 \mu \mathrm{J}$ and pulse durations $<15 \mathrm{fs}$ can be delivered at a high repetition rate. The IR laser is synchronized to the pulse pattern of the FLASH and presently runs at a repetition rate of $100 \mathrm{kHz}$ in $800 \mu \mathrm{s}$ bursts, repeating every $100 \mathrm{~ms}$ (further specifications can be found in Ref. [24]). The IR laser is coupled into the FEL beamline under 90 degrees by a silver-coated planar mirror. At this position, the FEL is focused down to less than $3 \mathrm{~mm}$ in diameter and passes through a $4 \mathrm{~mm}$ hole in this incoupling mirror. From this point on, the FEL and the IR beam travel collinearly. An IR-focussing lens with a focal length of $50 \mathrm{~cm}$ is placed outside the vacuum. Spatial overlap is set by means of a Ce:YAG-powder coated screen in the focal plane inside the REMI. The two foci of the FEL and the IR-laser on the screen can be observed with a CCD camera and magnifying optics [19]. Temporal overlap with nanosecond precision can be found with a fast photodiode downstream of the REMI. In order to exactly overlap the pulses in time, one uses a target with a well known pump-probe signal. The chosen target (e.g., helium, xenon) depends on the FEL 
photon energy and usually involves a specific excitation and a following ionization step which is just possible for the right temporal order of FEL and IR.

\subsection{HHG Radiation}

The setup of the HHG source is a result of a collaboration between MPIK, DESY and the university of Hanover [25]. The IR beam is transferred to a neighboring laser table where high harmonics in the XUV regime are generated in a noble-gas target [26]. The generated XUV light can be separated from the fundamental IR, either with an aluminum filter or a sufficiently small aperture, as the IR has a larger divergence than the XUV. First runs of the HHG source were successful and commissioning results can be found in Ref. [25]. The HHG-XUV light is coupled into the FEL beamline by a mirror under $8^{\circ}$ grazing incidence. The XUV beam has a vertical offset of $7.5 \mathrm{~mm}$ with respect to the FEL. In this configuration, each of the two light pulses (HHG and FEL) is reflected on a separate half of the split mirror and can be steered and delayed with respect to each other.

\subsection{Reaction Microscope}

With the reaction microscope [27] ionization and fragmentation processes of single atoms or molecules are studied. As depicted in Figure 2, laser beam and target gas jet are oriented orthogonally to each other and cross in the center of the REMI. A stack of metal rings produces a homogeneous electric field that separates positively and negatively charged particles and guides them onto the detectors. Helmholtz coils generate a homogeneous magnetic field forcing the electrons on a spiral trajectory to ensure large acceptance for electrons with large transversal momentum inside the spectrometer. Each of the two time- and position-sensitive detectors is composed of a set of micro channel plates and a delay line anode [28]. Time-of-flight information and the impact position on the detector allow to calculate the initial momentum at the time when the charged particle was created. Momentum conservation in three-dimension is used to sort out all particles originating from the same fragmenting target particle, that is finding coincident particles. Angle- and energy-resolved measurements of charged particles in multi-coincidence are the key features of the REMI and enable to investigate atomic and molecular processes in detail $[5,6,29,30]$.

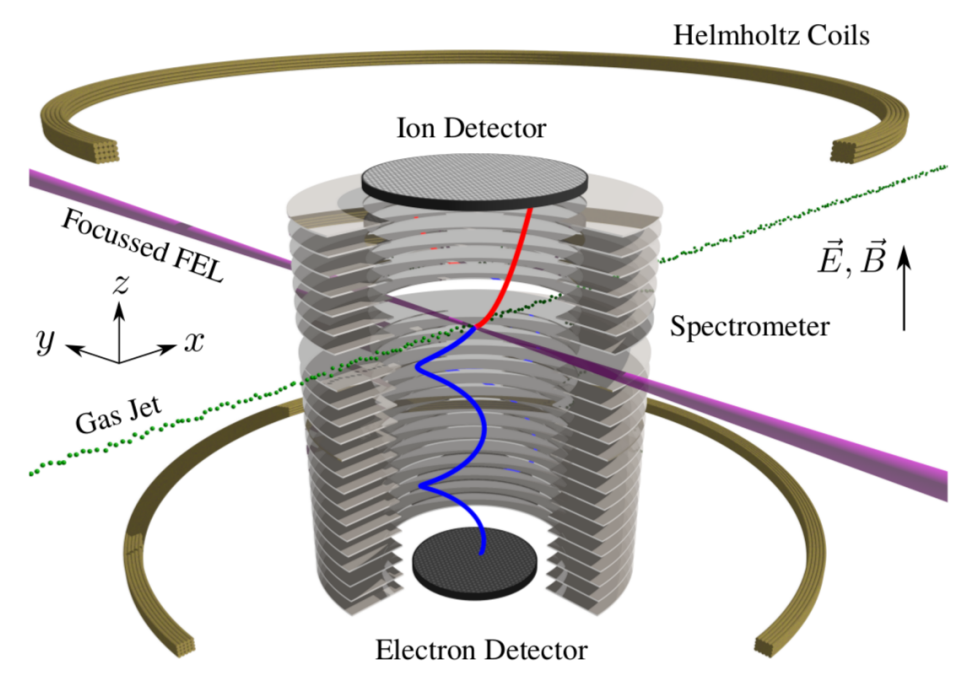

Figure 2. Schematic of the reaction microscope. Reproduced from [19], with the permission of the International Union of Crystallography. 


\section{Selected Results}

\subsection{Angle-Resolved Wavelength Scan: XUV+IR in Helium}

This experiment makes use of the wavelength tunability of FLASH 2 and the $4 \pi$ angular acceptance of the reaction microscope. As will be shown in the following, it demonstrates a beneficial combination of the FEL and the IR laser.

In this pump-probe measurement the XUV light of the FEL was used to excite helium, while a subsequent IR-laser pulse ionizes the excited atom. Scanning the XUV photon energy from $20.4 \mathrm{eV}$ to just below the ionization threshold of $24.6 \mathrm{eV}$ reveals the excitation transition series $1 s^{2} \rightarrow 1$ sn $p$ in helium [31], in accordance with the dipole selection rules for linearily polarized light. The excited helium atom absorbs multiple photons (each $1.55 \mathrm{eV}$ ) of the subsequent IR pulse which bring the electron to the continuum. This process is shown in Figure 3a, where an increased photoelectron yield is observed at XUV energies that matches the specific excitation energy $(1 s 2 p$ at $21.2 \mathrm{eV}, 1 \mathrm{~s} 3 p$ at $23 \mathrm{eV} \ldots)$. The distribution of the photoelectron kinetic energy is plotted along the ordinate and shows several peaks at specific XUV excitation energies. The underlying process is the above threshold ionization (ATI) and describes the behavior of an electron absorbing more photons than the minimal requirement to be emitted into the continuum [32,33]. The additional electron energy is directly related to the IR-photon energy (in this case $\approx 1.55 \mathrm{eV}$ ).

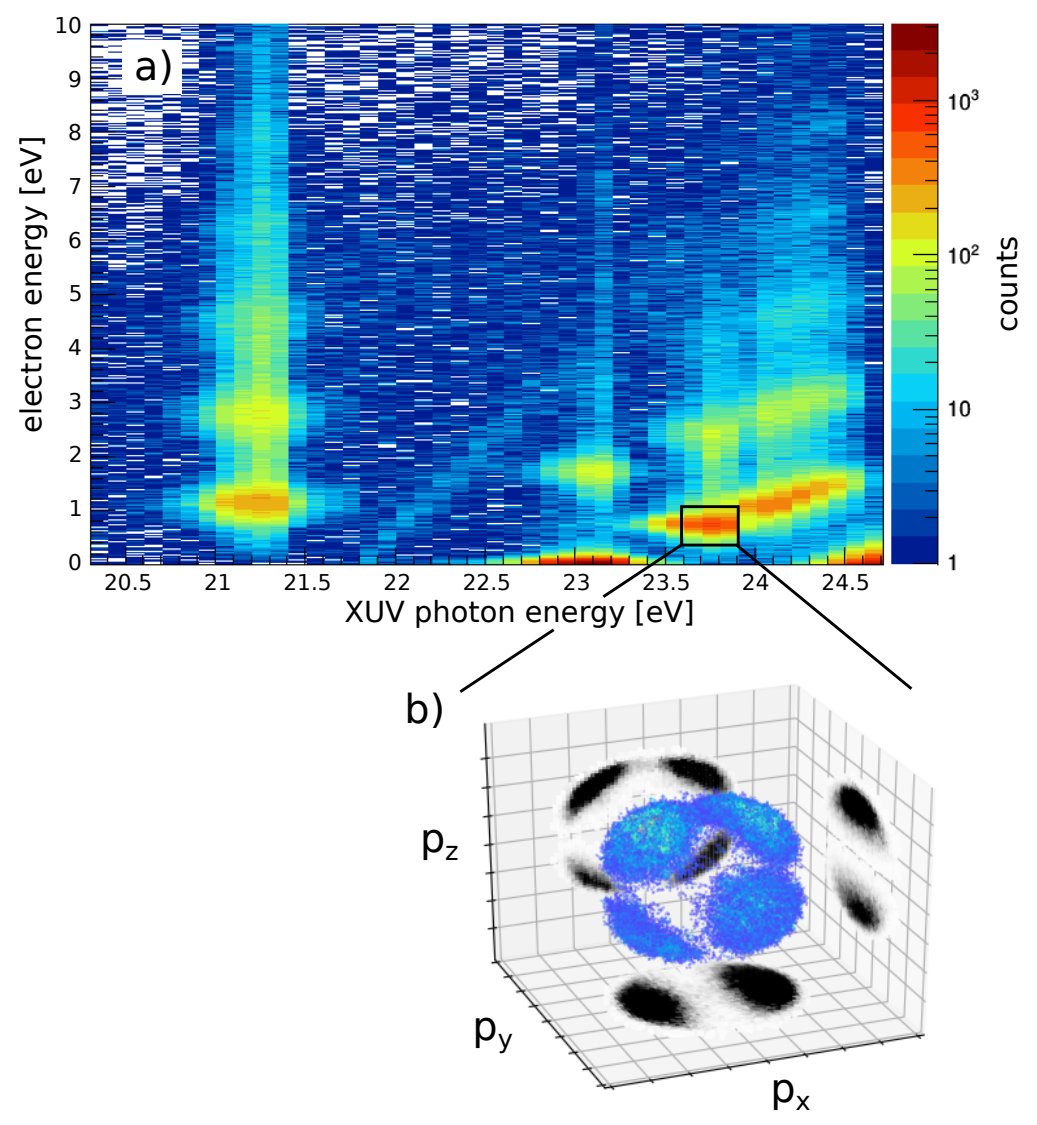

Figure 3. (a) XUV photon energy scan with delayed IR-laser pulse, plotted versus photoelectron kinetic energy. Resonances in helium are revealed for specific XUV energies and above threshold ionization (ATI) can be seen by the distribution of the photoelectron kinetic energy. (b) Electron momentum distribution at the specific region indicated by the black square. The XUV is polarized along $p_{x}$, the IR along $p_{z}$.

While in Figure 3a only the absolute electron kinetic energy is plotted, the REMI allows to measure the electron momentum in $3 \mathrm{D}$ as shown in Figure $3 \mathrm{~b}$. The XUV is polarized along the $p_{x}$ direction and 
the IR laser along the $p_{z}$ direction. Figure $3 \mathrm{~b}$ contains exclusively electrons recorded with parameters in the marked region of Figure 3a. First an XUV photon excites the helium atom into the $1 s 4 p$ state $(23.7 \mathrm{eV})$ and upon the additional absorption of one IR photon, the 4 p-electron is emitted into the continuum. Starting from the helium groundstate, the absorption of two photons can yield to an angular momentum of $l=2$ for the photoelectron, with the corresponding characteristic number of nodes in the angular distribution. The two orthogonal linearly polarized light fields can lead to the population of angular momentum substates with $m=0, \pm 2$. This can be understood as in a spherical basis each light field can be described by a superposition of left- and right-handed circulary polarized light, driving $\Delta m= \pm 1$ transitions. For a detailed explanation see [34].

This experiment demonstrates the capability to precisely measure electron angular distributions which is essential to investigate many different phenomena like quantum beating [35], electron correlation [36] or propensity rules [37].

\subsection{Two-Photon Double Ionization in Argon}

In a kinematically complete experiment, double ionization of argon by absorption of two photons was measured [30]. Neutral argon atoms were irradiated by FEL radiation with a photon energy of $27.93 \mathrm{eV}$ (averaged peak intensity $(3 \pm 2) \times 10^{13} \mathrm{~W} / \mathrm{cm}^{2}$, pulse duration approximately $50 \mathrm{fs}$ ). The angular distributions of the ejected photoelectrons during two-photon double ionization (TPDI) were measured. The dominant TPDI channel observed was sequential double ionization (SDI), in which both photoelectrons were emitted from the argon atom by sequential single-photon absorption (see also [38]). The two photoelectrons were confirmed to be correlated through polarization of the intermediate $\mathrm{Ar}^{+}$state. As a result, the measured angular distributions of both, the first and the second, photoelectron differ from that of the single ionization. In addition, the coincident detection method led to the discovery of the crucial role of autoinization in both steps of SDI in argon.

Both the ion and the electron detector of the REMI have multi-hit capability. This is necessary for the coincident detection of the two photoelectrons emerging from one TPDI event. Additionally, $\mathrm{Ar}^{+}$ions were measured in coincidence with the photoelectrons. This allows to differentiate between particles stemming from single ionization and the SDI channel. As both processes occur during the same experiment, the influence of systematic errors can be greatly reduced by comparing results from these two ionization pathways.

Figure 4 shows the two-dimensional kinetic energy spectrum of two photoelectrons detected in coincidence. Because the second ionization potential of argon lies just below the photon energy of the FEL radiation, there is one fast (first) and one slow (second) photoelectron expected during SDI [39]. This feature of correlated electrons in SDI is shown in Figure 4, where the kinetic energy of two photoelectrons created within one XUV pulse are plotted against each other. There is an increased yield of photoelectrons at positions where one photoelectron has a kinetic energy of $12 \mathrm{eV}$ and where the other electron has a kinetic energy of $0.3 \mathrm{eV}$. The increased photoelectron yield at $E_{1}=12 \mathrm{eV}$ and $E_{2}=12 \mathrm{eV}$ shows uncorrelated electrons and can be attributed to electrons from two independent single ionization processes. The capability to record such two-dimensional energy spectra of reaction fragments detected in coincidence underlines the aptitude of the REMI as a versatile AMO (atomic, molecular, and optical physics) science station. The dynamics following multi-photon absorption processes in the XUV range is kinematically completely accessible. 


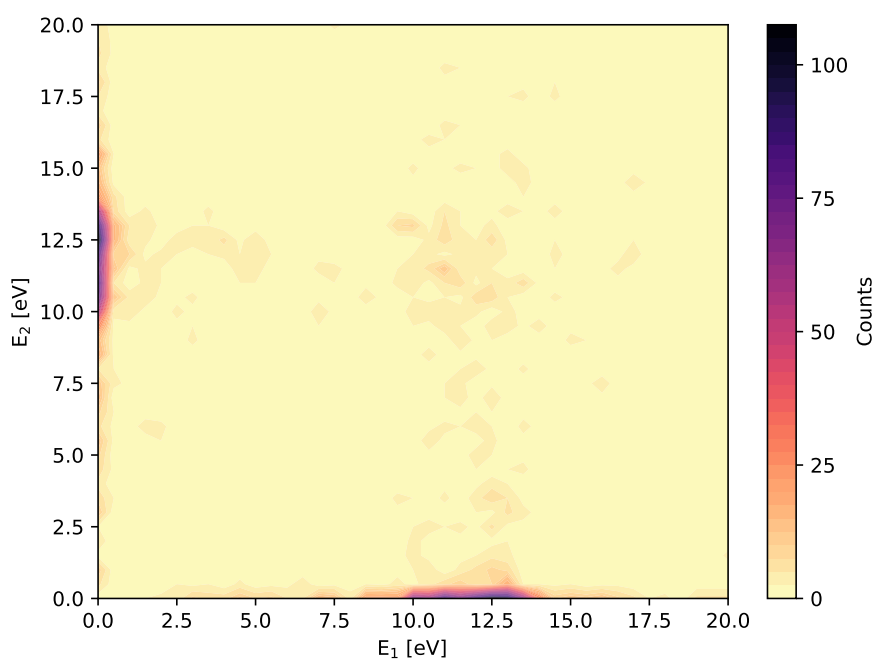

Figure 4. Two-dimensional kinetic energy spectrum of photoelectrons originating from the same laser pulse in two-photon double ionization (TPDI) in argon. The clustering at low $E_{1}$ and low $E_{2}$ are the signature of the sequential double ionization (SDI) channel. The blob on the diagonal is caused by false coincidences. Reproduced and modified from [30].

\subsection{Molecular Dynamics in Ar Dimers}

The pump-probe technique combined with fragment ion momentum imaging allows to track the evolution of intermediate states through observables in the final state as a function of the pump-probe delay [6].

The key observables to track molecular dynamics in REMI experiments are the yield of a specific coincidence channel and the kinetic energy release (KER) of this channel. The KER is the sum of the nuclear kinetic energies, which are generated from potential energy. While the system evolves along the potential energy curve of the intermediate state it accumulates the kinetic energy $\operatorname{KER}_{n l}\left(t_{d}\right)$, until it is probed at the time $t_{d}$ and at the internuclear distance $R\left(t_{d}\right)$. Then it accumulates further energy on the final state potential $K_{E} R_{f}\left(t_{d}\right)$ and we observe the sum $K E R\left(t_{d}\right)=K E R_{n l}\left(t_{d}\right)+K E R_{f}\left(t_{d}\right)$ (see Figure 5).

The capabilities to observe molecular dynamics with this setup is demonstrated exemplarily in an experiment investigating charge transfer (CT) in argon dimers using an XUV-pump IR-probe scheme [5]. The experiment was performed at FLASH1, using the same reaction microscope apparatus, but a different IR pump laser [40]. Argon dimers were ionized and excited to $\mathrm{Ar}^{2+*}-\mathrm{Ar}$ states by absorption of three $27 \mathrm{eV}$ photons. These states are still weakly bound, but the equilibrium nuclear distance is much smaller. So the nuclei start to move towards each other. At smaller internuclear distances, the potential energy curve crosses unbound states of the $\mathrm{Ar}^{+*}-\mathrm{Ar}^{+}$type which are coupled nonadiabatically so charge transfer can happen there (see Figure 5).

The system is subsequently probed by an intense $800 \mathrm{~nm}$ laser pulse [40] to the $\mathrm{Ar}^{2+}-\mathrm{Ar}^{+}$final state. This transition is only possible if charge transfer has taken place. Therefore the yield of this particular channel gives information on the transition probability of the charge transfer. In this process we actually have two intermediate states. The first can only contribute negligible kinetic energy as the potential is shallow. Thus the KER is accumulated mainly on the second intermediate state and the final state.

The value of interest is the time constant when the system transfers its charge and thus changes from the first to the second intermediate. Restricting the KER to values corresponding to the distance $R_{X}$, where crossings appear, allows to select those events that were probed directly after the charge is transferred (see Figure 6). With this restriction, the probe pulse directly tracks the transition rate depending on the time delay. This shows how the measurement of KERs combined with ion yields 
for varying pump-probe delays, allows to deduce the transition rates of the process of interest as a function of time.

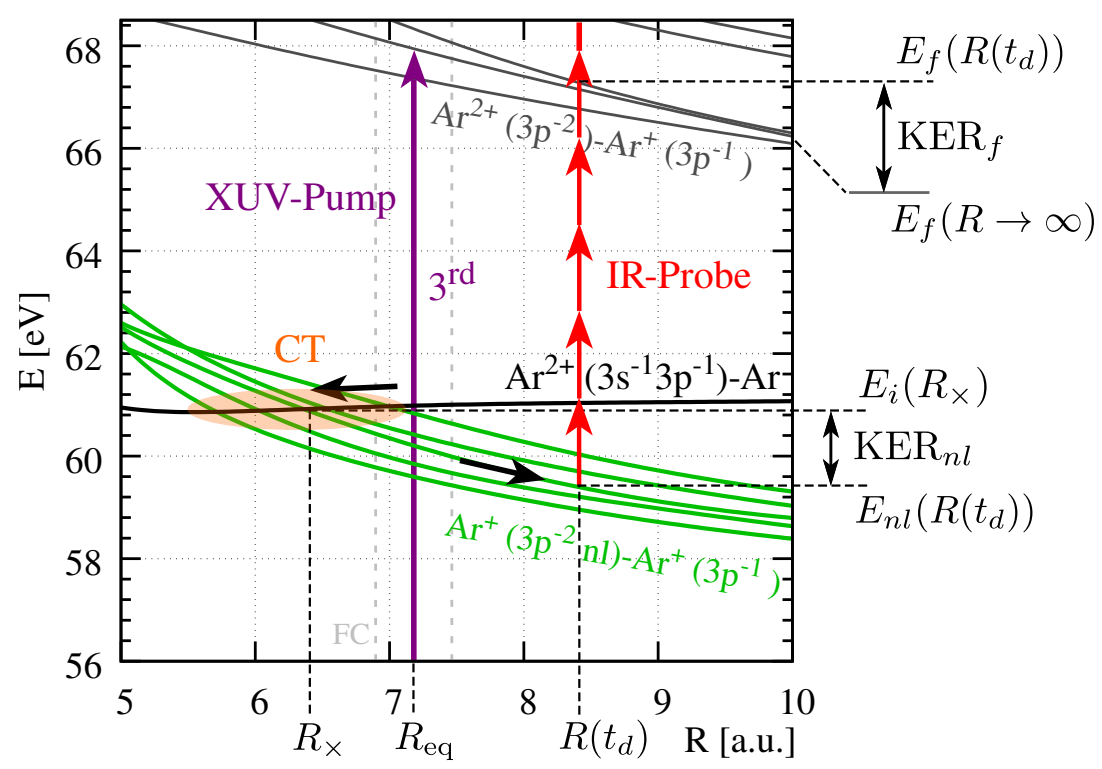

Figure 5. Potential energy curves of the argon dimer in the energy range $56 \mathrm{eV}-68.5 \mathrm{eV}$. The XUV pump (purple arrow) populates the initial $\mathrm{Ar}^{2+}\left(3 \mathrm{~s}^{-1} 3 \mathrm{p}^{-1}\right)-\mathrm{Ar}$ state (black curve) at $R_{\mathrm{eq}}$. The wave packet starts to evolve to smaller $R$. At the crossings (orange-shaded area) with $\operatorname{Ar}^{+}\left(3 \mathrm{p}^{-2} \mathrm{nl}\right)-\mathrm{Ar}^{+}\left(3 \mathrm{p}^{-1}\right)$ states (light-green curves), charge transfer (CT) happens. The IR probe (red arrows) interrogates the population of the $\operatorname{Ar}^{+}\left(3 p^{-2} n l\right)-\operatorname{Ar}^{+}\left(3 p^{-1}\right)$ states by ionization to final $\operatorname{Ar}^{2+}\left(3 p^{-2}\right)-\operatorname{Ar}^{+}\left(3 p^{-1}\right)$ states (dark-grey curves). Specific potential energies and corresponding internuclear distances are indicated by horizontal and vertical dashed lines, respectively. Reproduced from [5], with the permission of AIP (American Institute of Physics) Publishing.

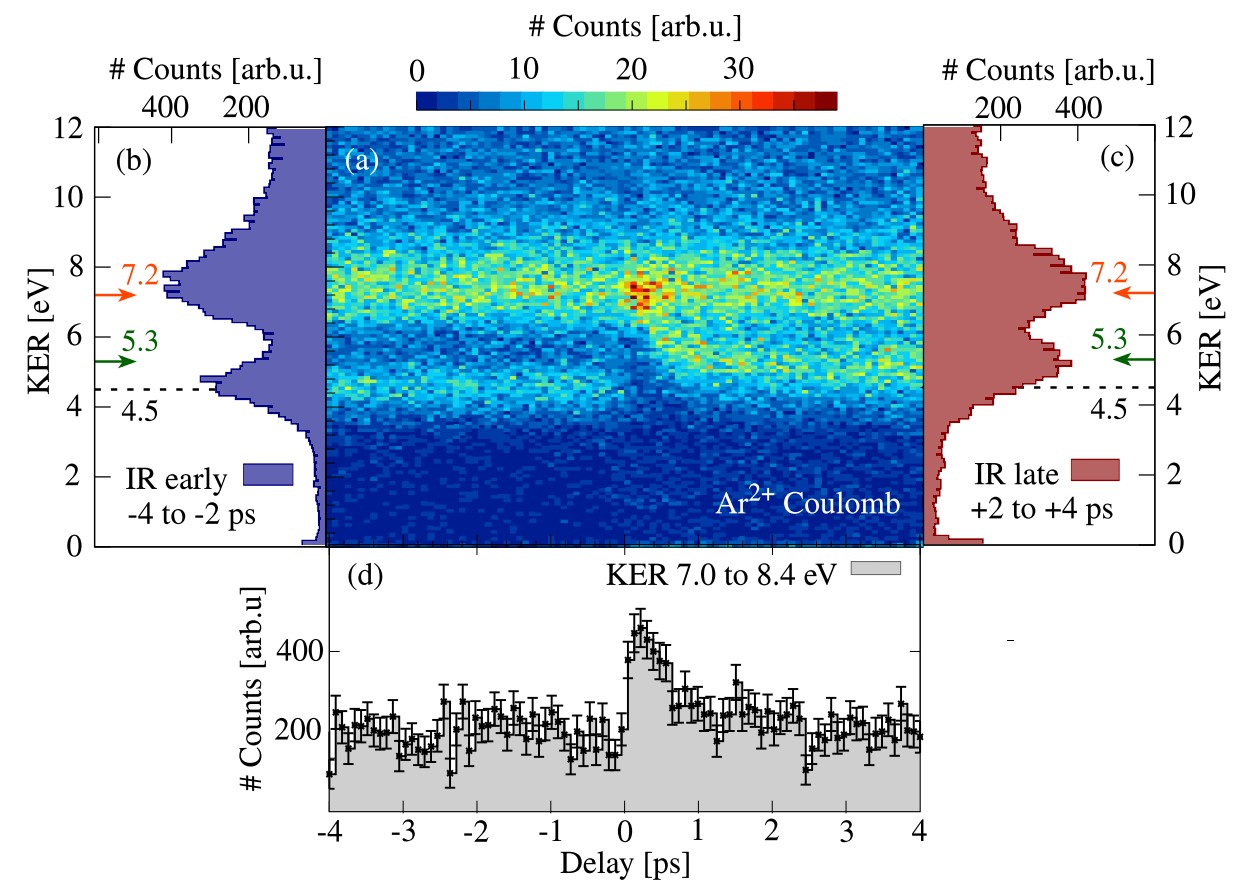

Figure 6. Coulomb-exploded $\mathrm{Ar}^{2+}$ ions (non-coincident). (a) KER (kinetic energy release) vs. delay. For negative delays, the IR is early with respect to the XUV pulse, for positive delays vice versa. (b) Projection of all KERs within -4 to -2 ps onto the KER axis. (c) Same as (b), but for +2 to +4 ps. (d) Projection of all KERs between 7.0 to $8.4 \mathrm{eV}$ onto the delay axis. Reproduced from [5], with the permission of AIP Publishing. 


\section{Conclusions}

We have presented the REMI endstation at FLASH2. The unique combination of a reaction microscope and three state-of-the-art light sources opens up the possibilities for unprecedented investigations in the field of atomic and molecular physics.

The sophisticated detection scheme of the REMI enables to measure charged fragments' momenta in 3D and in a coincident manner, which makes it an outstanding tool to extract essential information of atomic and molecular fragmentation processes. We reviewed three experiments, each pointing out specific strengths of the setup. The first experiment in Section 3.1, the XUV-pump IR-probe measurement in helium showed the capability of scanning the XUV energy of FLASH2 and the 3D momentum resolution of the REMI. The second experiment in Section 3.2 on the two-photon double ionization in argon revealed the necessity of a coincident measurement to sort out electrons stemming from specific channels. The third experiment in Section 3.3 on argon dimers showed, how to investigate dynamical processes like charge migration by means of delay-dependent KER analysis.

Besides XUV-XUV and XUV-IR experiments, future experiments aim for pump-probe experiments with the already commissioned HHG source in combination with the FEL. A future extension of the endstation is an XUV spectrometer that will be installed downstream of the REMI. The spectrometer allows to analyze FEL-pulses on a shot to shot basis, which can be used to sort REMI data according to the fluctuating SASE-generated pulses.

The REMI endstation at FLASH2 facilitates many collaborations with a large variety of experiments. Future proposals for experiments are already accepted and planned.

Author Contributions: Conceptualization, S.M., F.T., H.L. and R.M.; Investigation S.M., F.T., H.L., R.M., K.S., S.A., M.B. and T.P.; Writing-original draft preparation S.M., F.T., H.L. and R.M.; writing-review and editing, S.M., F.T., H.L., S.A., K.S., T.P., R.T., M.B. and R.M.; Visualization, S.A. and S.M.; Project administration, R.T., T.P. and R.M. All authors have read and agreed to the published version of the manuscript.

Funding: This research received no external funding.

Conflicts of Interest: The authors declare no conflict of interest.

\section{References}

1. Strickland, D.; Mourou, G. Compression of amplified chirped optical pulses. Opt. Commun. 1985, 55, 447-449. [CrossRef]

2. Zewail, A.H. Laser Femtochemistry. Science 1988, 242, 1645-1653. [CrossRef] [PubMed]

3. Ullrich, J.; Rudenko, A.; Moshammer, R. Free-Electron Lasers: New Avenues in Molecular Physics and Photochemistry. Annu. Rev. Phys. Chem. 2012, 63, 635-660. [CrossRef] [PubMed]

4. Jiang, Y.H.; Senftleben, A.; Kurka, M.; Rudenko, A.; Foucar, L.; Herrwerth, O.; Kling, M.F.; Lezius, M.; Tilborg, J.V.; Belkacem, A.; et al. Ultrafast dynamics in acetylene clocked in a femtosecond XUV stopwatch. J. Phys. B At. Mol. Opt. Phys. 2013, 46, 164027. [CrossRef]

5. Schmid, G.; Schnorr, K.; Augustin, S.; Meister, S.; Lindenblatt, H.; Trost, F.; Liu, Y.; Miteva, T.; Gisselbrecht, M.; Düsterer, S.; et al. Tracing charge transfer in argon dimers by XUV-pump IR-probe experiments at FLASH. J. Chem. Phys. 2019, 151, 084314. [CrossRef]

6. Schnorr, K.; Senftleben, A.; Kurka, M.; Rudenko, A.; Schmid, G.; Pfeifer, T.; Meyer, K.; Kübel, M.; Kling, M.F.; Jiang, Y.H.; et al. Electron Rearrangement Dynamics in Dissociating $\mathrm{I}_{2}^{n+}$ Molecules Accessed by Extreme Ultraviolet Pump-Probe Experiments. Phys. Rev. Lett. 2014, 113, 073001. [CrossRef]

7. Jiang, Y.H.; Rudenko, A.; Herrwerth, O.; Foucar, L.; Kurka, M.; Kühnel, K.U.; Lezius, M.; Kling, M.F.; van Tilborg, J.; Belkacem, A.; et al. Ultrafast Extreme Ultraviolet Induced Isomerization of Acetylene Cations. Phys. Rev. Lett. 2010, 105, 263002. [CrossRef]

8. Orzechowski, T.J.; Anderson, B.; Fawley, W.M.; Prosnitz, D.; Scharlemann, E.T.; Yarema, S.; Hopkins, D.; Paul, A.C.; Sessler, A.M.; Wurtele, J. Microwave radiation from a high-gain free-electron laser amplifier. Phys. Rev. Lett. 1985, 54, 889-892. [CrossRef] 
9. Emma, P.; Akre, R.; Arthur, J.; Bionta, R.; Bostedt, C.; Bozek, J.; Brachmann, A.; Bucksbaum, P.; Coffee, R.; Decker, F.J.; et al. First lasing and operation of an ångstrom-wavelength free-electron laser. Nat. Photonics 2010, 4, 641-647. [CrossRef]

10. Ackermann, W.A.; Asova, G.; Ayvazyan, V. Operation of a free-electron laser from the extreme ultraviolet to the water window. Nat. Photonics 2007, 1, 336-342. [CrossRef]

11. Brabec, T.; Krausz, F. Intense few-cycle laser fields: Frontiers of nonlinear optics. Rev. Mod. Phys. 2000, 72, 545-591. [CrossRef]

12. Cerullo, G.; Baltuška, A.; Mücke, O.; Vozzi, C. Few-optical-cycle light pulses with passive carrier-envelope phase stabilization. Laser Photonics Rev. 2011, 5, 323-351. [CrossRef]

13. Corkum, P.B. Plasma perspective on strong field multiphoton ionization. Phys. Rev. Lett. 1993, 71, $1994-1997$. [CrossRef] [PubMed]

14. Lewenstein, M.; Balcou, P.; Ivanov, M.Y.; L'Huillier, A.; Corkum, P.B. Theory of high-harmonic generation by low-frequency laser fields. Phys. Rev. A 1994, 49, 2117-2132. [CrossRef]

15. McPherson, A.; Gibson, G.; Jara, H.; Johann, U.; Luk, T.S.; McIntyre, I.A.; Boyer, K.; Rhodes, C.K. Studies of multiphoton production of vacuum-ultraviolet radiation in the rare gases. J. Opt. Soc. Am. B 1987, 4, 595-601. [CrossRef]

16. Calegari, F.; Sansone, G.; Stagira, S.; Vozzi, C.; Nisoli, M. Advances in attosecond science. J. Phys. B At. Mol. Opt. Phys. 2016, 49, 062001. [CrossRef]

17. Vozzi, C.; Calegari, F.; Frassetto, F.; Poletto, L.; Sansone, G.; Villoresi, P.; Nisoli, M.; De Silvestri, S.; Stagira, S. Coherent continuum generation above $100 \mathrm{eV}$ driven by an IR parametric source in a two-color scheme. Phys. Rev. A 2009, 79, 033842. [CrossRef]

18. Boutet, S.; Yabashi, M. X-Ray Free Electron Lasers and Their Applications. In X-ray Free Electron Lasers: A Revolution in Structural Biology; Springer International Publishing: Cham, Switzerland, 2018; pp. 1-21. [CrossRef]

19. Schmid, G.; Schnorr, K.; Augustin, S.; Meister, S.; Lindenblatt, H.; Trost, F.; Liu, Y.; Braune, M.; Treusch, R.; Schröter, C.D.; et al. Reaction microscope endstation at FLASH2. J. Synchrotron Radiat. 2019, 26, 854-867. [CrossRef]

20. Ayvazyan, V.; Baboi, N.; Bähr, J.; Balandin, V.; Beutner, B.; Brandt, A.; Bohnet, I.; Bolzmann, A.; Brinkmann, R.; Brovko, O.I.; et al. First operation of a free-electron laser generating GW power radiation at $32 \mathrm{~nm}$ wavelength. Eur. Phys. J. D At. Mol. Opt. Plasma Phys. 2006, 37, 297-303. [CrossRef]

21. Faatz, B.; Plönjes, E.; Ackermann, S.; Agababyan, A.; Asgekar, V.; Ayvazyan, V.; Baark, S.; Baboi, N.; Balandin, V.; von Bargen, N.; et al. Simultaneous operation of two soft X-ray free-electron lasers driven by one linear accelerator. New J. Phys. 2016, 18, 062002. [CrossRef]

22. Faatz, B.; Braune, M.; Hensler, O.; Honkavaara, K.; Kammering, R.; Kuhlmann, M.; Ploenjes, E.; Roensch-Schulenburg, J.; Schneidmiller, E.; Schreiber, S.; et al. The FLASH Facility: Advanced Options for FLASH2 and Future Perspectives. Appl. Sci. 2017, 7, 1114. [CrossRef]

23. Deutsches Elektronen-Synchrotron DESY. FLASH Parameters. Available online: https://photon-science. desy.de/facilities/flash/flash_parameters/index_eng.html (accessed on 9 March 2020).

24. Lang, T.; Alisauskas, S.; Große-Wortmann, U.; Hülsenbusch, T.; Manschwetus, B.; Mohr, C.; Müller, J.; Peters, F.; Schirmel, N.; Schulz, S.; et al. Versatile OPCPA Pump-Probe Laser System for the FLASH2 XUV FEL Beamline at DESY. In Proceedings of the 2019 Conference on Lasers and Electro-Optics Europe European Quantum Electronics Conference (CLEO/Europe-EQEC), Munich, Germany, 23-27 June 2019; p. 1. [CrossRef]

25. Appi, E.; Papadopoulou, C.; Mapa, J.; Wesavkar, N.; Jusko, C.; Mosel, P.; Alisauskas, S.; Lang, T.; Heyl, C.; Manschwetus, B.; et al. A synchronized VUV light source based on high-order harmonic generation at FLASH. Sci. Rep. 2020, 10, 6867. [CrossRef]

26. Heyl, C.M.; Arnold, C.L.; Couairon, A.; L'Huillier, A. Introduction to macroscopic power scaling principles for high-order harmonic generation. J. Phys. B At. Mol. Opt. Phys. 2016, 50, 013001. [CrossRef]

27. Ullrich, J.; Moshammer, R.; Dorn, A.; Dörner, R.; Schmidt, L.P.H.; Schmidt-Böcking, H. Recoil-ion and electron momentum spectroscopy: reaction-microscopes. Rep. Prog. Phys. 2003, 66, 1463-1545. [CrossRef]

28. Jagutzki, O.; Dangendorf, V.; Lauck, R.; Czasch, A.; Milnes, J. A position- and time-sensitive photon-counting detector with delay- line read-out. Opt. Sens. Technol. Appl. 2007. [CrossRef] 
29. Kurka, M.; Rudenko, A.; Foucar, L.; Kühnel, K.U.; Jiang, Y.H.; Ergler, T.; Havermeier, T.; Smolarski, M.; Schössler, S.; Cole, K.; et al. Two-photon double ionization of Ne by free-electron laser radiation: A kinematically complete experiment. J. Phys. B At. Mol. Opt. Phys. 2009, 42, 141002. [CrossRef]

30. Augustin, S.; Schulz, M.; Schmid, G.; Schnorr, K.; Gryzlova, E.V.; Lindenblatt, H.; Meister, S.; Liu, Y.F.; Trost, F.; Fechner, L.; et al. Signatures of autoionization in the angular electron distribution in two-photon double ionization of Ar. Phys. Rev. A 2018, 98, 033408. [CrossRef]

31. National Institute of Standards and Technology (NIST). NIST Atomic Spectra Database Levels Data. Available online: https:/ / physics.nist.gov / cgi-bin /ASD / energy1.pl?encodedlist=XXT2\&de=0\&spectrum= $\mathrm{He}+\mathrm{I} \&$ submit=Retrieve + Data\&units $=1 \&$ format $=0 \&$ output $=0 \&$ page_size $=15 \&$ multiplet_ordered $=0 \&$ conf out=on\&term_out=on\&level_out=on\&unc_out=1\&j_out=on\&lande_out=on\&perc_out=on\&biblio=on \& temp $=($ accessed on 16 April 2020).

32. Agostini, P.; Fabre, F.; Mainfray, G.; Petite, G.; Rahman, N.K. Free-Free Transitions Following Six-Photon Ionization of Xenon Atoms. Phys. Rev. Lett. 1979, 42, 1127. [CrossRef]

33. Corkum, P.B.; Burnett, N.H.; Brunel, F. Above-threshold ionization in the long-wavelength limit. Phys. Rev. Lett. 1989, 62, 1259-1262. [CrossRef]

34. Kerbstadt, S.; Pengel, D.; Johannmeyer, D.; Englert, L.; Bayer, T.; Wollenhaupt, M. Control of photoelectron momentum distributions by bichromatic polarization-shaped laser fields. New J. Phys. 2017, 19, 103017. [CrossRef]

35. Fechner, L.; Camus, N.; Ullrich, J.; Pfeifer, T.; Moshammer, R. Strong-Field Tunneling from a Coherent Superposition of Electronic States. Phys. Rev. Lett. 2014, 112, 213001. [CrossRef]

36. Fechner, L.; Camus, N.; Krupp, A.; Ullrich, J.; Pfeifer, T.; Moshammer, R. Creation and survival of autoionizing states in strong laser fields. Phys. Rev. A 2015, 92, 051403. [CrossRef]

37. Busto, D.; Vinbladh, J.; Zhong, S.; Isinger, M.; Nandi, S.; Maclot, S.; Johnsson, P.; Gisselbrecht, M.; L'Huillier, A.; Lindroth, E.; et al. Fano's Propensity Rule in Angle-Resolved Attosecond Pump-Probe Photoionization. Phys. Rev. Lett. 2019, 123, 133201. [CrossRef] [PubMed]

38. Braune, M.; Hartmann, G.; Ilchen, M.; Knie, A.; Lischke, T.; Reinköster, A.; Meissner, A.; Deinert, S.; Glaser, L.; Al-Dossary, O.; et al. Electron angular distributions of noble gases in sequential two-photon double ionization. J. Mod. Opt. 2016, 63, 324-333. [CrossRef]

39. Grum-Grzhimailo, A.N.; Gryzlova, E.V.; Strakhova, S.I.; Kabachnik, N.M.; Fritzsche, S. Angular distributions and correlations in sequential two-photon atomic double ionization. J. Phys. Conf. Ser. 2009, 194, 012004. [CrossRef]

40. Redlin, H.; Al-Shemmary, A.; Azima, A.; Stojanovic, N.; Tavella, F.; Will, I.; Düsterer, S. The FLASH pump-probe laser system: Setup, characterization and optical beamlines. Nucl. Instrum. Methods Phys. Res. Sect. A Accel. Spectrom. Detect. Assoc. Equip. 2011, 635, S88-S93. [CrossRef] 